\title{
CNS Spectrums: a new publisher, a new editor-in-chief, and new vistas
}

With this issue of CNS Spectrums, a new era begins. First published in 1996 and fully indexed in 2003, CNS Spectrums has just been acquired by Cambridge University Press and will now join Cambridge's distinguished collection of journals in psychiatry and mental health, such as Behavioral and Brain Sciences, Psychological Medicine, International Journal of Neuropsychopharmacology (published on behalf of the International College of Neuropsychopharmacology-CINP), the Journal of the International Neuropsychological Society, and many more (see http://journals.cambridge.org/psych).

As the newly appointed Editor-in-Chief, I am tasked now with the reorganization and relaunch of CNS Spectrums over the course of 2012, during which time we will reconfigure the editorial board, produce four quarterly issues, and refocus the aims and scope of the journal, from that of mostly original articles to an emphasis on short, illustrated reviews of topics with special relevance to readers and investigators in clinical neuroscience specialties. In particular, we aim to commission and attract reviews and opinion pieces in translational neuroscience, biological psychiatry, and neuropsychopharmacology that explain clinically relevant neuroscience discoveries in a way that makes these findings accessible and understandable to clinicians and clinical investigators. We will emphasize new therapeutics of all types in clinical neurosciences, mental health, psychiatry, and neurology, especially first in man studies and proof of concept studies. Our focus will be not just drugs, but as demonstrated in this first issue of 2012, novel psychotherapies and neurostimulation therapeutics as well. Original research will continue to be published alongside reviews. In the short run, in fact, we will publish mostly original articles coming from the journal's current pipeline of submissions, but aim to include more reviews and opinion pieces by the end of the year, and to expand back to monthly issues over time.
We are pleased to announce as well that CNS Spectrums is now the official journal of the Neuroscience Education Institute (NEI), and that NEI members will receive free online access to the journal as part of their membership (see http://neiglobal. com). You will note that the abstracts from the 7th Annual NEI Psychopharmacology Congress, held in November of 2011, are included in this first issue of 2012, and CNS Spectrums will publish these every year, including the abstracts of posters presented at the upcoming 8th Annual NEI Psychopharmacology Congress to be held in San Diego in October 2012.

Moving forward, CNS Spectrums will be accessible to library and consortia customers through the Cambridge collections and subscription services. Throughout 2012, CNS Spectrums will be offered free of charge to current institutional libraries throughout the world. We plan to distribute some special issues and CME supplements in print and online over the next year and beyond, and we expect to announce some additional digital offerings in the near future.

So, it is with great excitement that we bring these changes to the attention of our readers and contributors and look forward to your comments and suggestions as we relaunch, rebrand, and re-energize CNS Spectrums. I would like to extend my personal thanks and those of Cambridge University Press to the past editors, reviewers, and authors who have contributed to the current status of the journal, and without whom we would not be relaunching with such high hopes of a continued bright future.

Stephen M. Stahl, MD, PhD Editor-in-Chief Department of Psychiatry University of California San Diego, San Diego, California and Department of Psychiatry University of Cambridge, Cambridge, UK 